\title{
Effectiveness of Progestogens as Maintenance Tocolysis and Urogenital Cultures: Secondary Analysis of the PROTECT Trial
}

\author{
Fabio Facchinetti, MD ${ }^{1}$ \\ ${ }^{1}$ Department of Mother Infant, Obstetrics and Gynecology Unit, \\ University of Modena and Reggio Emilia, Modena, Italy \\ 2 Department of Diagnostic, Clinical and Public Health Medicine, Statistics \\ Unit, University of Modena and Reggio Emilia, Modena, Italy \\ ${ }^{3}$ Department of Medicine and Surgery, Obstetrics Gynecology Branch, \\ University of Milano-Bicocca Health Science, Milano, Lombardia, Italy \\ ${ }^{4}$ Department of Health Science, Obstetrics and Gynecology Branch, \\ University of Florence, Florence, Italy \\ ${ }^{5}$ Obstetrics and Gynecology Unit, Istituto di Ricovero e Cura a \\ Carattere Scientifico Policlinico, Milan, Italy \\ ${ }^{6}$ Department of Surgical Sciences, Gynaecology and Obstetrics, \\ University of Turin, Turin, Piemonte, Italy \\ Am J Perinatol Rep 2020;10:e198-e201.
}

Lucrezia Pignatti, MD ${ }^{1}$ Roberto D’Amico, $\mathrm{PhD}^{2}$ Patrizia Vergani, MD ${ }^{3}$ Mariarosaria Di Tommaso, MD, $\mathrm{PhD}^{4}$ Barbara Acaia, MD ${ }^{5}$ Chiara Benedetto, MD, $\mathrm{PhD}^{6}$

\begin{abstract}
Address for correspondence Lucrezia Pignatti, MD, Division of Maternal Fetal Medicine, Mother Infant Department, University of Modena and Reggio Emilia, Via del Pozzo 71, 41124 Modena, Italy (e-mail: lucrezia.pignatti@gmail.com).
\end{abstract}

\begin{abstract}
Keywords

- preterm labor

- progestogens

- maintenance tocolysis

- genitourinary infection

Background In a recently published multicenter randomized controlled trial, we demonstrated that progestogens are not effective as maintenance tocolysis.

Objective This study was aimed to evaluate if previous finding may be affected by positive urine culture and/or vaginal swab.

Study Design We performed a secondary analysis of the PROTECT trial (NCT01178788). Women with singleton pregnancy between 22 and $31^{6 / 7}$ weeks' gestation, admitted for threatened preterm labor were considered. At admission, we collected urine culture and vaginal swabs. At discharge, women with a cervical length $\leq 25 \mathrm{~mm}$ were randomized to vaginal progesterone or 17 $\alpha$-hydroxyprogesterone caproate or observation group. We used Chi-square statistics, considering $97.5 \% \mathrm{Cl}$ (confidence interval) and $p$-value less than 0.025 for significance.

Results Urine culture and vaginal swabs were collected in 232 out of 235 patients included in the primary analysis. Overall, 31 out of 232 women (13.4\%) had positive urine culture and 60 out of 232 (25.9\%) had positive vaginal swab. In women with negative urine culture, a higher rate of preterm birth was found in vaginal progesterone group $(27 / 69,39.7 \%)$ respect with controls $(14 / 68,20.6 \%$; relative risk $[R R]=1.90$; 97.5\% Cl: $1.01-3.57 ; p=0.018)$.

Conclusion Among women with negative urine culture, the rate of preterm birth $<37$ weeks' gestation was significantly increased in those receiving vaginal progesterone, reinforcing our previous findings in symptomatic women.
\end{abstract}

received

September 3, 2019 accepted after revision April 6, 2020
DOI https://doi.org/

10.1055/s-0040-1713788. ISSN 2157-6998.
Copyright $\odot 2020$ by Thieme Medical

Publishers, Inc., 333 Seventh Avenue, New York, NY 10001, USA. Tel: $+1(212) 760-0888$.
License terms

$\Theta(1) \Theta \circledast$ 
Preterm birth is a leading cause of perinatal morbidity and mortality, and in developed Countries, it occurs in the 6 to $11 \%$ of pregnancies. About two-thirds of preterm births are spontaneous, following spontaneous onset of labor or subsequent to preterm premature rupture of membranes. ${ }^{1}$

Preterm birth is a syndrome, and multiple etiologies contribute to the premature activation of one or more of the components of the pathways of parturition. Intrauterine infection is one of them, perhaps accounting for 25 to $40 \%$ of preterm birth. The most common pathways of access to the amniotic cavity is the ascending route from the vagina and the cervix. ${ }^{1,2}$ Asymptomatic bacteriuria and symptomatic urinary tract infections significantly increase the rates of preterm delivery, also by activating the inflammatory cascade. ${ }^{3,4}$ Bacterial vaginosis has also been associated with a two-fold increase in risk of preterm birth, although treatment did not reduce the risk. , $6^{5}$

Progestogens are one of the few interventions able to prevent preterm birth, in selected cases. However, we recently compared $17 \alpha$-hydroxyprogesterone caproate, vaginal progesterone, and no treatment as maintenance tocolysis after a threatened preterm labor episode in singletons with no history of preterm birth. In the primary analysis of the trial, neither $17 \alpha$-hydroxyprogesterone caproate nor vaginal progesterone was effective. Moreover, treatment with vaginal progesterone seemed to increase the risk of preterm birth before 35 weeks, and in those women with cervical length between 15 and $25 \mathrm{~mm}^{7}$

The aim of our study was to perform a secondary analysis, to evaluate if our finding may be affected by other factors, namely, the presence of a positive urine culture and/or positive vaginal swab.

\section{Materials and Methods}

This is a secondary analysis of the PROTECT trial (NCT01178788), a multicenter, open-label, randomized controlled trial performed in five tertiary level University hospitals in Northern Italy, already approved by the local Ethics Committee. ${ }^{7}$ Women with singleton pregnancies between 22 and $31^{6 / 7}$ weeks of gestation, who had been admitted to the hospital because of their first threatened preterm labor episode was considered.

At admission, for every woman, we performed urine culture and vaginal swabs. A midstream urine sample was collected in a sterile urine container. Urine culture was performed by manual calibrated loop technique. Quantitative cultures of urine were performed for the detection of uropathogens on $5 \%$ sheep blood Columbia agar, Chromogenic media CPS, and Candida Id plate (bioMerieux). Vaginal specimens were plated into appropriate agar mediums: chocolate, MacConkey, 5\% sheep blood, Gardnerella agar, Candida Id, StreptoB plates, and enrichment broths (Brain heart Infusion broth medium and thioglycolate broth medium; bioMerieux). Symptomatic positive urine culture and positive vaginal swabs were treated according to bacteriological sampling and susceptibility tests. In each center, different antibiotic regimens were used accordingly to local protocols. Penicillin (ampicillin, amoxicillin, and amoxicillin clavulanic acid), cefalosporin (cefixime and ceftriaxone), macrolides (azithromycin, erythromycin, and josamycin), and metronidazole were the prescribed antibiotics.

At discharge, women who had a cervical length $\leq 25 \mathrm{~mm}$ were randomly assigned to receive a once-a-week intramuscular injection of $341 \mathrm{mg} 17 \alpha$-hydroxyprogesterone caproate (17P group) or vaginal administration of $200 \mathrm{mg}$ daily of micronized progesterone (P group), or no treatment (Control group). Treatments were stopped at 36 completed weeks of gestation.

Data were collected by each participant center in an online database and statistical analysis were performed at the Statistic Unit of the University of Modena and Reggio Emilia. For the secondary analysis we used Chi-square statistics, considering a $97.5 \% \mathrm{CI}$ (confidence interval) and a $p$ value of less than 0.025 for significance to take into account multiple comparisons.

\section{Results}

Between July 2010 and June 2015, 257 women were found eligible to participate in the PROTECT study. ${ }^{7}$ Three of them declined to participate and 254 were subsequently randomly assigned to the three arms. Demographic characteristic did not differ between groups in terms of maternal age, ethnicity, body mass index, smoking during pregnancy, low education, parity, previous spontaneous miscarriages, gestational age at enrolment, and cervical length. ${ }^{7}$ Women with transvaginal ultrasound cervical length less than $15 \mathrm{~mm}$ at enrolment were equally distributed in the three groups: $21 \%$ in $17 \alpha-$ hydroxyprogesterone caproate group, $32 \%$ in vaginal progesterone group, and $30 \%$ in controls. ${ }^{7}$

Finally, urine culture and vaginal swabs were collected in 232 out of 235 patients included in the primary analysis. Out of these 232 patients, 78 received $17 \alpha$-hydroxyprogesterone caproate, 77 received vaginal progesterone, and 77 represented the control group.

Overall, 31 out of 232 women (13.4\%) had positive urine culture and 60 out of 232 (25.9\%) had a positive vaginal swab. The distribution of positive tests was similar between groups (-Table 1). In urine cultures, the most frequent isolates were Escherichia coli (15, 6.5\%), Streptococcus agalactiae (9, 3.9\%), and Enterococcus faecalis (6, 2.6\%). Among vaginal swabs, the most common isolates were $S$. agalactiae (26, 11.2\%),

Table 1 Positive cultures among groups

\begin{tabular}{|l|l|l|l|l|}
\hline & $\begin{array}{l}\text { 17P } \\
\boldsymbol{n}=78(\%)\end{array}$ & $\begin{array}{l}\mathrm{P} \\
\boldsymbol{n}=77(\%)\end{array}$ & $\begin{array}{l}\text { Control } \\
\boldsymbol{n}=\mathbf{7 7}(\%)\end{array}$ & -Value \\
\hline $\begin{array}{l}\text { Positive } \\
\text { urine } \\
\text { culture }\end{array}$ & $14(17.9)$ & $8(10.4)$ & $9(11.7)$ & NS \\
\hline $\begin{array}{l}\text { Positive } \\
\text { vaginal } \\
\text { swab }\end{array}$ & $22(28.2)$ & $17(22.1)$ & $21(27.3)$ & NS \\
\hline
\end{tabular}

Abbreviations: 17P, 17 $\alpha$-hydroxyprogesterone caproate group; NS, not significant; $P$, vaginal progesterone group. 


\section{PTB and negative urine culture}

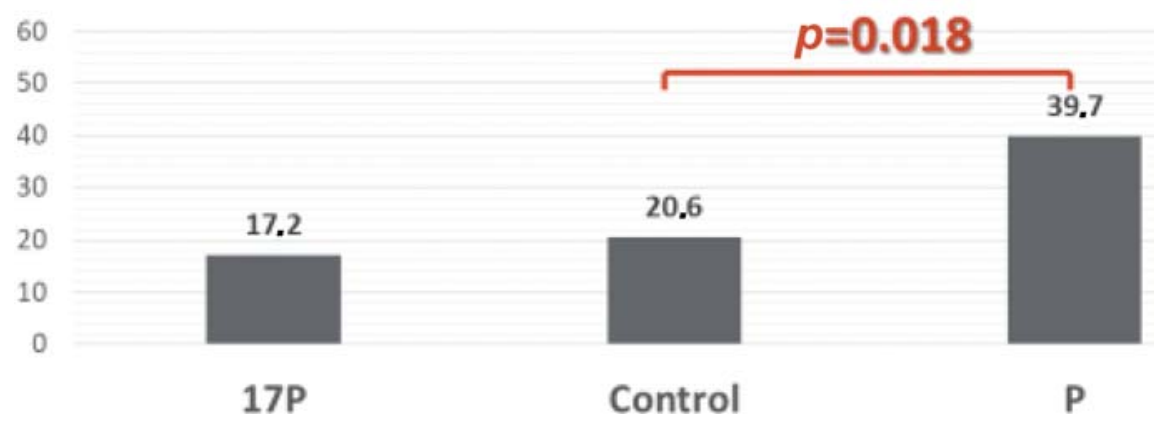

Fig. 1 Rate of preterm birth among women with negative urine culture in the three groups. PTB, preterm birth.

Ureaplasma urealyticum (18, 7.8\%), E. coli (6, 2.6\%), and Gardnerella vaginalis (6, 2.6\%).

Considering the whole sample, 12 women with positive urine culture delivered before 37 weeks (12/31, 38.7\%), not different to those with negative urine culture (52/201, $25.9 \%$ ). Twenty women with positive vaginal swab had a preterm birth $(20 / 60,33.3 \%)$, similar to ones with negative vaginal swab (44/172, 25.6\%).

In women with negative urine culture, a higher rate of preterm birth was found in those treated with vaginal progesterone $(27 / 69,39.7 \%)$ respect with controls $(14 / 68$, 20.6\%; relative risk $[\mathrm{RR}]=1.90 ; 97.5 \% \mathrm{CI}: 1.01-3.57$; $p=0.018 ;$ - Fig. 1 ). In women with positive urine culture, the rate of preterm birth was similar in all three groups and no correlation was found between vaginal swabs and the primary outcome comparing the three groups ( - Table 2 ).

\section{Discussion}

In this secondary analysis, we found that among women with negative urine culture, the rate of preterm birth $<37$ weeks' gestation was significantly increased in those receiving vaginal progesterone. No changes were observed in those exposed to $17 \alpha$-hydroxyprogesterone caproate.

Previous studies aimed to assess the efficacy of progestogens as maintenance tocolysis provided conflicting results. In a recent meta-analysis, authors described a possible positive effect of vaginal progesterone treatment in term of reduction of preterm birth rate and prolongation of pregnancy. However, given the lack of blinding in some trials and the generally poor quality of the trials, the authors do not suggest a change in clinical care of women with arrested preterm labor. ${ }^{8}$ Further, a more recent meta-analysis by Palacio et al found no differences in preterm birth rate or latency period in women treated with progestogens once; only high-quality studies were considered. ${ }^{9}$ In light of our findings, it is possible that the lack of urine culture evaluation could explain heterogeneity in the findings of previously considered primary trials.

The association between genitourinary tract infections in pregnancy and the risk of preterm birth has been repeatedly demonstrated. ${ }^{3-6}$ Asymptomatic bacteriuria is present in up to $10 \%$ of pregnant women, and it increases by two-fold the risk of preterm delivery. ${ }^{10,11}$ Accordingly, antibiotic treatment for asymptomatic bacteriuria and urinary tract infections reduces the risk of preterm birth and low-birth weight, ${ }^{10}$ and screening and treatment of asymptomatic bacteriuria become a standard of care. ${ }^{10-12}$

On the other hand, several cervicovaginal infections have been associated with preterm birth. These include ureaplasma colonization, trichomoniasis, and bacterial vaginosis. This condition is present in almost $20 \%$ of pregnant women, remaining asymptomatic in most cases. ${ }^{5-13}$ However, although bacterial vaginosis has been associated with a twofold increase in the risk of preterm birth, specific antibiotic treatments only allowed eradication without affecting the risk of premature birth. ${ }^{6}$

The reasons why vaginal progesterone is associated with increased preterm birth in symptomatic women remaining undelivered after preterm labor are unknown. One could only refer to cervical inflammation processes which have been repeatedly reported in the above condition. ${ }^{2-14}$

Table 2 Rate of PTB $<37$ weeks among women with positive and negative cultures in the three groups

\begin{tabular}{|l|l|l|l|l|l|l|l|l|}
\hline \multicolumn{2}{|c|}{} & \multicolumn{2}{|l|}{ PTB } & \multicolumn{1}{l|}{ 17P vs. control } & \multicolumn{2}{l|}{ P vs. control } \\
\cline { 3 - 9 } & $\begin{array}{l}17 P \\
n(\%)\end{array}$ & $\begin{array}{l}\text { P } \\
n(\%)\end{array}$ & $\begin{array}{l}\text { Control } \\
n(\%)\end{array}$ & RR (97.5\% Cl) & $p$-Value & RR (97.5\% Cl) & $p$-Value \\
\hline \multirow{2}{*}{$\begin{array}{l}\text { Urine } \\
\text { culture }\end{array}$} & Positive & $6 / 14(42.9)$ & $3 / 8(37.5)$ & $3 / 9(33.3)$ & $1.29(0.36-4.55)$ & 0.648 & $1.13(0.26-4.90)$ & 0.858 \\
\cline { 2 - 9 } & Negative & $11 / 64(17.2)$ & $27 / 69(39.1)$ & $14 / 68(20.6)$ & $0.83(0.37-1.88)$ & 0.618 & $1.90(1.01-3.57)$ & 0.018 \\
\hline \multirow{2}{*}{$\begin{array}{l}\text { Vaginal } \\
\text { swab }\end{array}$} & Positive & $7 / 22(31.8)$ & $8 / 17(47.1)$ & $5 / 21(23.8)$ & $1.33(0.44-4.10)$ & 0.558 & $1.98(0.69-5.64)$ & 0.133 \\
\cline { 2 - 9 } & Negative & $10 / 56(17.9)$ & $22 / 60(36.7)$ & $12 / 56(21.4)$ & $0.83(0.35-1.97)$ & 0.634 & $1.71(0.86-3.41)$ & 0.072 \\
\hline
\end{tabular}

Abbreviations: $\mathrm{Cl}$, confidence interval; P, vaginal progesterone group; PTB, preterm birth; RR, relative risk; 17p, 17 $\alpha$-hydroxyprogesterone caproate group. 
The presence of pathogens in the urogenital tract could excite an infection-based inflammatory status through activation of the innate immune system and proinflammatory cytokines production. Progesterone has immunomodulatory functions and modify tool-like receptors (TLRs) signaling, leading to a diminished macrophage response to stimuli and decreased cytokines expression. ${ }^{15}$ Moreover, it has been demonstrated that vaginal administration of micronized progesterone significantly reduces metalloproteinase expression and activity in fetal membranes stimulated with lipopolysaccharide, suggesting a possible protective mechanism of micronized progesterone in preventing infection-associated preterm birth. ${ }^{16}$

In our secondary analysis, we found an increased rate of preterm birth in women without urogenital tract colonization. It is possible that a different starting etiology is involved in such cases, and that the effects of progesterone may also depend on the mechanisms initiating inflammatory reactions. ${ }^{15}$

\section{Limitation}

A limitation of this secondary analysis is the lack of external validity.

\section{Conclusion}

In conclusion, the findings of this secondary analysis therefore suggest that the presence of urinary tract colonization/infection could represent a confounding factor for trials assessing the efficacy of preterm birth prevention. In our primary study the negative effect of vaginal progesterone in terms of increasing the risk of preterm birth, seems not related to the presence of the risk factors evaluated in this secondary analysis. Given the lack of information on the inflammatory status, we cannot define the possible causative mechanism underlying the different responses to vaginal progesterone administration in case of positive or negative urine culture. The evaluation of microbiological features and inflammatory milieu as confounding factors needs to be considered in future trials assessing the effectiveness of therapeutic options for the prevention of preterm birth.

\section{Conflict of Interest}

The authors report no conflict of interest.

\section{References}

1 Goldenberg RL, Culhane JF, Iams JD, Romero R. Epidemiology and causes of preterm birth. Lancet 2008;371(9606):75-84

2 Romero R, Espinoza J, Kusanovic JP, et al. The preterm parturition syndrome. BJOG 2006;113(Suppl 3):17-42

3 Kalinderi K, Delkos D, Kalinderis M, Athanasiadis A, Kalogiannidis I. Urinary tract infection during pregnancy: current concepts on a common multifaceted problem. J Obstet Gynaecol 2018;38(04): 448-453

4 Sheiner E, Mazor-Drey E, Levy A. Asymptomatic bacteriuria during pregnancy. J Matern Fetal Neonatal Med 2009;22(05): 423-427

5 Garland SM, Ní Chuileannáin F, Satzke C, Robins-Browne R. Mechanisms, organisms and markers of infection in pregnancy. J Reprod Immunol 2002;57(1,2):169-183

6 Cunnington M, Kortsalioudaki C, Heath P. Genitourinary pathogens and preterm birth. Curr Opin Infect Dis 2013;26(03): 219-230

7 Facchinetti F, Vergani P, Di Tommaso M, et al. Progestogens for maintenance tocolysis in women with a short cervix: a randomized controlled trial. Obstet Gynecol 2017;130(01): 64-70

8 Suhag A, Saccone G, Berghella V. Vaginal progesterone for maintenance tocolysis: a systematic review and metaanalysis of randomized trials. Am J Obstet Gynecol 2015;213(04): 479-487

9 Palacio M, Ronzoni S, Sánchez-Ramos L, Murphy KE. Progestogens as maintenance treatment in arrested preterm labor: a systematic review and meta-analysis. Obstet Gynecol 2016;128(05):989-1000

10 Smaill FM, Vazquez JC. Antibiotics for asymptomatic bacteriuria in pregnancy. Cochrane Database Syst Rev 2015;(08):CD000490

11 Romero R, Oyarzun E, Mazor M, Sirtori M, Hobbins JC, Bracken M. Meta-analysis of the relationship between asymptomatic bacteriuria and preterm delivery/low birth weight. Obstet Gynecol 1989;73(04):576-582

12 National Institute for Health and Care Excellence. Antenatal care for uncomplicated pregnancies. Available at: https://www.nice. org.uk/guidance/cg62. Accessed April 20, 2020

13 Pararas MV, Skevaki CL, Kafetzis DA. Preterm birth due to maternal infection: causative pathogens and modes of prevention. Eur J Clin Microbiol Infect Dis 2006;25(09):562-569

14 Romero R, Espinoza J, Gonçalves LF, Kusanovic JP, Friel L, Hassan S. The role of inflammation and infection in preterm birth. Semin Reprod Med 2007;25(01):21-39

15 Larsen B, Hwang J. Progesterone interactions with the cervix: translational implications for term and preterm birth. Infect Dis Obstet Gynecol 2011;2011:353297

16 Hung TH, Chen SF, Wu CP, Li MJ, Yeh YL, Hsieh TT. Micronized progesterone pretreatment affects the inflammatory response of human gestational tissues and the cervix to lipopolysaccharide stimulation. Placenta 2017;57:1-8 